Research Article

\title{
Application of Nanosystems Synergized by Optics and Chemotherapy in the Treatment of Sports Rehabilitation Diseases
}

\author{
Zhiwei Peng, ${ }^{1,2}$ Xianxiong Li ${ }^{1},{ }^{1}$ and Yu Zhang ${ }^{1}$ \\ ${ }^{1}$ School of Physical Education, Hunan Normal University, Changsha 410012, Hunan, China \\ ${ }^{2}$ Department of Physical Education and Research, Hunan University of Technology and Business, Changsha 410205, \\ Hunan, China \\ Correspondence should be addressed to Xianxiong Li; lixianxiong@hunnu.edu.cn
}

Received 7 August 2020; Revised 9 September 2020; Accepted 14 October 2020; Published 30 October 2020

Academic Editor: Tifeng Jiao

Copyright (c) 2020 Zhiwei Peng et al. This is an open access article distributed under the Creative Commons Attribution License, which permits unrestricted use, distribution, and reproduction in any medium, provided the original work is properly cited.

\begin{abstract}
With the rapid development of sports in my country, many athletes will have mild or severe diseases. Physical rehabilitation can improve their physical pain, and some more serious diseases require optical and chemotherapy treatments. This article mainly studies the application of nanosystems that cooperate with optical and chemotherapy in the treatment of sports rehabilitation diseases. In the experiment, the bacteria-infected mice in the wound were divided into 5 groups $(n=4)$, and the mouse wound model was made. After that, the number of bacteria remaining in the wound was determined by the plate count method. At the same time, a cytotoxicity test was performed, and a control experiment was performed on the cell culture of the polymer at a concentration below the VCR and CMC values. The final sections were used for tissue analysis of serine and epoxy staining. It was found from the experimental data that the cumulative release of free CLB molecules within 3 hours almost reached 100\%, and its rapid release was attributed to its small molecular size. In contrast, the release of CLB-HDH micelles in $3 \mathrm{~h}$ is $62 \%$, which has a certain relaxation effect. The results showed that CLB-HDH micelles significantly inhibited tumor growth, had good safety in vivo, and had low systemic toxicity. CLB-HDH micelles provide new ideas for efficient CLB tumor treatment.
\end{abstract}

\section{Introduction}

With the rapid development of nanotechnology, nanomaterials have also developed rapidly with the development of biotechnology. The application of nanomaterials in the medical field has opened up new areas for the development of biosensors, imaging technologies, and target carriers, especially in the treatment of sports rehabilitation disorders. Phototherapy combined with chemotherapy is an effective way to optimize the treatment of sports rehabilitation disorders. In the coordinated system of phototherapy and chemotherapy, the improvement in vascular permeability induced by phototherapy increases the accumulation of nanomedicine, which can be used to enhance the effect of chemotherapy. In addition, chemotherapy can inhibit the regeneration of damaged blood vessels and target the remaining cancer cells, enhancing the effect of phototherapy. However, most of the currently commonly used chemotherapeutics and photosensitizers are hydrophobic and do not target tumors. Direct administration will reduce the therapeutic effect and cause serious toxicity. When the nanocarrier is applied to the phototherapy and chemotherapy system in coordination, the nanocarrier is attached to the phototherapeutic agent and the chemotherapy agent at the same time, which increases the accumulation of the therapeutic agent on the tumor and reduces side effects.

The construction of a biologically effective nanomedicine system is a new research direction for antitumor target drugs. In other words, it can improve the antitumor activity of antitumor compounds, improve the biocompatibility and stability of drugs, improve tumor specificity, and reduce drugs. Placing the drug inside the MSN or adsorbing it on the surface can increase the effective concentration of the drug, increase the utilization rate of the drug, and reduce the toxicity and side effects during drug transportation. In addition, the surface modification of MSN 
also includes folic acid (FA), aniline (Tf), hyaluronic acid (HA), tumor cell membrane, and other active target recognition groups, which can improve the delivery and recognition of active targets at the tumor site of the nanoparticles.

Light therapy and chemotherapy have the effect of enhancing treatment and reducing side effects in clinical animal studies. Soares et al. believe that severe play (SG) has been used to improve physical activity, balance training, and muscle strength in elderly people with frailty syndrome (FS). The purpose of this research is to evaluate the effect of an exercise plan that uses the SG developed for the rehabilitation of the elderly. In this quasi-experimental study, they divided 24 institutionalized seniors (16 women) with a mean (SD) age of 80.9 (5.6) years into two groups (experimental group and control group). The SG exercise program is performed twice a week for three months (20 sessions, 15-25 minutes each). They evaluated participants' mobility in the Timekeeping and Walking Test (TUG), the balance of the Functional Reach Test (FRT), and the strength of the gripper and upper and lower limb dynamometer. Although this research has certain reference significance, it lacks experimental data [1].

Spagnoli proposed a camera-based method for identifying patients and detecting the interaction between patients and therapists during treatment. He believes that detecting interactions can help distinguish between active and passive movements of patients and estimate the accuracy of bone data. He uses continuous face recognition to detect, identify, and track patients with other people in the scene (such as therapists or clinicians). He uses a method based on local binary pattern (LBP). After he determines the user in the scene, he will determine the interaction between the patient and other people. He uses depth maps/point clouds to estimate the distance between two people. Although his research is feasible in theory, it is difficult to operate [2].

Dana believes that neuromuscular damage and reduced musculoskeletal integrity are signs of spinal cord injury (SCI) that hinders exercise recovery. He believes that these injuries are caused by nerve damage and lead to abuse, which has aroused interest in activity-based physical rehabilitation $(\mathrm{ABT})$, which can promote neuromuscular plasticity after SCI. However, as the severity of SCI increases, the efficacy of ABT decreases. In addition, many men with SCI show lower testosterone levels, which may exacerbate neuromusculoskeletal damage. He believes that the incorporation of testosterone adjuvants and ABT may improve musculoskeletal recovery and neuroplasticity because androgen attenuates muscle loss after SCI and the transition of muscle fiber types from slow to fast in a manner independent of mechanical strain and promotes the survival of motor neurons. Although testosterone alone produces only limited functional improvement in rodent SCI models, these neuromusculoskeletal benefits are promising. His research is not rigorous enough [3].

In this paper, the nanodrug delivery system is used to realize the common carrier of photosensitizer and anticancer agent, improve the stimulation of photosensitizer and anticancer agent to tumor, and increase the accumulation of tumor site. Various organic nanodrug delivery systems, such as reposom, missel, dendritic cells, and hollow polymer cells, can be effectively used for PS and chemotherapeutic drug delivery. In addition, a series of inorganic nanocarriers, such as zinc oxide, graphene, carbon materials, and quantum dots, are also used as cocarriers of photosensitizers and anticancer agents. In this paper, polymer micelles with navigation molecules on the surface were used as transport carriers, which effectively increased the concentration of therapeutic drugs in multidrug-resistant cells so that multidrug-resistant cells could resensitize drugs.

\section{Nanosystem with Synergy of Optics and Chemotherapy}

2.1. Nanosystem. The size of nanoparticles is larger than clusters of atoms and smaller than usual particles, generally in the range of 1 nanometer to 100 nanometers. In other words, nanoparticles are fine particles that are invisible to the naked eye and ordinary microscopes. Nanoparticles can reach tumor tissues only when they penetrate the blood vessel wall and tumor matrix, and their particle size must be consistent with the pore size of the capillary wall. If the particle size is too large or too small, the EPR effect cannot be obtained. Only when it is close to the capillary pore, it will leak from the capillary and stay in the tumor tissue. The size of nanomaterials is restricted to several directions, and there is no periodicity. The energy balancer equation adds boundary conditions to create quantum size effects. The energy band, wave function, charge density, and state density of nanomaterials are different from all distributions. In bulk materials, the electron density is limited by size, forming a quantitative part. The quantum well is formed in a nano-superlattice, and the energy band is the energy level or subband of the nanomaterial [4].

Compared with traditional drugs, nanodrug carriers have many advantages: small particle size and large specific surface area. With small particle size and large specific surface area, nano drug carriers can improve the stability and solubility of drugs by loading or adsorbing drug molecules through covalent bonds, hydrogen bonds, and hydrophobic interactions. Nanodrug carriers transport drugs to lesions through human physiological barriers and are absorbed by cells through the terminal nervous system, which can increase the absorption rate of cellular drugs, reduce the frequency and dosage of drug delivery, and reduce cell drug resistance, toxicity, and side effects [5].

The formation of nanomicelles is related to the synthesis of ideal amphiphilic block copolymers and the formation of micelles under critical micelle concentration (CMC) conditions. The techniques for synthesizing amphiphilic copolymers usually include methods such as ring-opening polymerization, ATRP polymerization, and RAFT polymerization [6]. Absorption band gap is calculated as follows:

$$
E(r)=E_{g}(r=\infty)+\frac{h^{2} \pi^{2}}{2 \mu r^{2}}-\frac{1.786 e^{2}}{\varepsilon r}-0.248 E_{R y} .
$$


In the formula, $E(r)$ is the absorption band gap of the nanoparticle and $E_{g}(r=\infty)$ is the band gap of the bulk phase.

The relationship between scattered light intensity and particle size is as follows:

$$
I_{\theta}=\frac{9 \pi^{2} c v^{2}}{2 \lambda^{4} R^{2}} I_{0} \cdot\left(\frac{n_{2}^{2}-n_{1}^{2}}{n_{2}^{2}+2 n_{1}^{2}}\right)\left(1+\cos ^{2} \theta\right) .
$$

In the formula, $I_{\theta}$ is the intensity of scattered light in the $\theta$ direction and $R$ is the distance from the detector to the sample.

The CMC value determines the thermodynamic stability of the micelles. In the application of polymeric micelles, the micelle solution will be infinitely diluted by the circulating blood after intravenous injection, so the thermodynamic stability is very important. If the concentration of polymer forming micelles is lower than CMC, the micelles will be destroyed as soon as possible. When the target is reached, the loaded drug will be released into the circulation system. In this way, insoluble drugs will deposit on blood vessels, causing some toxic side effects. On the contrary, due to the interpenetration of hydrophilic chains, the concentration of copolymers should not exceed the range of aggregation or precipitation of micelles. Therefore, during drug delivery, each polymer has an appropriate concentration range to meet the requirements [7]. The calculation formula of the $\mathrm{CMC}$ value is as follows:

$$
\begin{aligned}
E(A) & =\frac{1}{n} \sum_{i=1}^{n} \frac{\min \left\{\mu \bar{A}\left(x_{i}\right), v \bar{A}\left(x_{i}\right)\right\}+\min \left\{\mu \stackrel{+}{A}\left(x_{i}\right), v \stackrel{+}{A}\left(x_{i}\right)\right\}+\pi \bar{A}\left(x_{i}\right)+\pi \stackrel{+}{A}\left(x_{i}\right)}{\max \left\{\mu \bar{A}\left(x_{i}\right), v \bar{A}\left(x_{i}\right)\right\}+\max \left\{\mu \stackrel{+}{A}\left(x_{i}\right), v \stackrel{+}{A}\left(x_{i}\right)\right\}+\pi \bar{A}\left(x_{i}\right)+\pi \stackrel{+}{A}\left(x_{i}\right)} \\
f\left(x_{1}, y_{1}, x_{2}, y_{2}\right) & =\frac{e^{1-z_{1}}\left(1-x_{1}\right)+e^{y_{1}} y_{1}}{e^{1-z_{1}}+e^{y_{1}}}+\frac{e^{1-z_{2}}\left(1-x_{2}\right)+e^{y_{2}} y_{2}}{e^{1-z_{2}}+e^{y_{2}}} .
\end{aligned}
$$

\subsection{Phototherapy and Chemotherapy}

2.2.1. Light Therapy. The nano-photoresponsive agent solves the problem of the dissolution and concentration of the photoresponsive agent, realizes the accumulation of tumor tissue, and can achieve good biocompatibility and joint diagnosis and treatment functions. The photosensitizers commonly used in tumor photodynamic therapy include metal complexes, BODIPY, bis-Ruinin, and princesin. The reaction mechanism of many photosensitizers is type II, which is strongly dependent on oxygen. However, the hypoxic nature of the tumor reduces the local $\mathrm{O}_{2}$ concentration of the tumor, and the hypoxic nature limits the effect of PDT. The photosensitizer accumulated in the target tissue, oxygen in the tissue, and light of a specific wavelength are the three conditions necessary for photodynamic therapy [8].

The biological mechanism of PDT in tumor treatment can be summarized from the following three aspects:

(1) Kill tumor cells directly. The ROS produced by PDT has a very short existence time and low diffusion and mobility, and its range of action is limited. Therefore, PDT causes oxidative damage to biomolecules such as proteins, lipids, and nucleic acids in the photosensitive area. The various ways to guide cell death depend on the location of the photosensitizer in the cell: damage to mitochondria may induce cell death; loss of cell membrane structure and integrity may lead to cell necrosis; damage to cells by small organs such as resom or vesicles may cause automatic death.

(2) Damage to the vascular system of tumor tissue. The normal survival of tissue cells depends on the supply of nutrients to blood vessels, so killing the blood vessel system at the tumor site is a strategy to treat tumor diseases.

(3) Strengthen the antitumor immune response. Molecular cytology studies have shown that the PDT treatment of solid tumors causes local inflammation, which can allow immune cells such as Mclovac, lymphocytes, and neutrophils to enter the tumor site in large quantities and promote various immunomodulators such as IL- 6 and IL-10. The production of prostaglandins, white blood cell walking factors, etc. will attract more immune cells to tumor tissues [9].

Photothermal therapy is a method of irradiating abundant light-to-heat conversion materials with near-infrared rays at the tumor site to effectively convert the absorbed light energy into heat energy, and the local temperature rises to cause tumor cell death. When the temperature rises to $42-45^{\circ} \mathrm{C}$, the cell will activate the protease, thereby destroying the mitochondria and causing reversible damage to the cell. When the temperature exceeds $50^{\circ} \mathrm{C}$, the cell membrane will be destroyed, and protein denaturation will cause irreversible damage to the cell. High temperature will destroy the structure of proteins and nucleic acids in the human body, thereby killing tumor cells. The local high temperature generated by PTT will increase the transparency of the cell membrane, directly affecting the absorption of inorganic salt ions (potassium, sodium, and calcium ions), some proteins, and adenosine triphosphate. Moreover, it can also promote chemotherapy agents to invade cells and strengthen the damage to cells. High temperature cannot directly damage the DNA chain in the nucleus, but it will cause changes in the structure of the chromotropic protein 
and hinder its synthesis. PTT may increase the DNA damage in the cell and affect the DNA repair process. The high temperature generated by PTT will damage the normal bones of cells, affect cell function, and eventually cause cell death. Moreover, the microenvironment of the tumor site has the characteristics of low oxygen and acidity. Cells in this state are more sensitive to heat, which helps high temperature kill tumor cells $[10,11]$.

On the one hand, when chemotherapeutic drugs are used to treat tumor cells, antitumor drugs penetrate into tumor cells, effectively kill them and reduce the volume of tumor cells, increase the sensitivity of tumor cells to cytotoxic T-lymphocyte specific killing, promote tumor cells, and express and release immunogenic substances. Removal of immunosuppressive cells will change the immunosuppressive state of the tumor microenvironment. On the other hand, immunotherapy enhances the sensitivity of cancer patients to chemotherapy by enhancing the body's immune system and antitumor immune response [12].

At present, almost no chemotherapy is new, but its effect has been improved because it can be introduced into the cancer site in a more effective way than before. Compared with taking a large number of drugs for a long time, it is better to maintain the effective concentration of drugs for a long time and have fewer side effects. For drugs inherent in the circulation, since cells can always reach the most effective stage of drugs, the delivery time is long, which has the advantage of fixing cells in different stages. Sometimes, the drug does not enter the blood circulation, but directly into the affected area. Alkylation reagents and DNA bases form additional substances that hinder DNA synthesis. Almost all alkylation reagents have two functional groups, which react with DNA bases to form intrastrand and lock bridges on two DNA strands. These crosslinks can be formed at any stage of the cell cycle, so alkylating agents are not phase specific. Metabolic antagonists inhibit nucleic acid synthesis, and their actual mechanism depends on the metabolic antagonists themselves [13].

2.3. Sports Rehabilitation. Sports rehabilitation training is not to let the disabled patients passively receive treatment, but in essence, it is the patients themselves actively exercising to achieve the purpose of treatment. However, this does not mean that patients must carry out exercise and training according to their own will, but according to the characteristics and severity of the disease or injury, as well as the functional status of patients with disabilities, by selecting appropriate physical rehabilitation methods, formulating corresponding exercise prescriptions, and carrying out under the guidance and supervision of medical personnel and sports rehabilitation training experts [14].

\section{Experiments of Nanosystems in Disease Diagnosis and Treatment}

3.1. Experimental Reagents and Experimental Instruments. The main reagents and instruments used in the experiment are shown in Tables 1 and 2 .

\subsection{Solution Preparation}

(1) Preparation of amino MSN (MSN-NH

Weigh $0.5 \mathrm{~g}$ CTAB $(1.35 \mathrm{mmol})$ into a round-bottom flask, add $250 \mathrm{ml}$ deionized water, and drop $1.77 \mathrm{ml}$ $\mathrm{NaOH}$. After stirring and heating to raise the temperature of the $\mathrm{CTAB}$ solution to $75^{\circ} \mathrm{C}$, drop $2.4 \mathrm{ml}$ TEOS. After continuing the reaction for 2 hours, the resulting precipitate was centrifuged at $9000 \mathrm{rpm}$ for 6 minutes to obtain white powder of MSN. Then, the product was dispersed in methanol solution containing $0.7 \mathrm{~mL}$ concentrated hydrochloric acid (37.2\%) and refluxed for 6 hours to remove the template CTAB from the particle pores. $\mathrm{Msn}-\mathrm{NH}_{2}$ was obtained by adding $0.5 \mathrm{~g}$ MSN into $40 \mathrm{ml}$ dry toluene, dropping $0.5 \mathrm{ml}$ of attes and reacting for 12 hours [15].

(2) Preparation of allyl block blocking polymer

First, in a flame-dried reaction flask, $4 \mathrm{ml}$ of potassium naphthalene solution and $0.5 \mathrm{ml}$ of foliol were stirred for 15 minutes. Then, under the protection of argon, a certain amount of tetrahydrofuran solution is transferred. After stirring for 15 minutes, place the reaction bottle in an ice salt bath and cool to $-5^{\circ} \mathrm{C}$. The premeasured $\mathrm{EO}$ gas passes through the drying device slowly through the reaction bottle for the anion reaction. Three days after the conversion of ethylene oxide to peg, a certain amount of a-cl monomer was injected under the protection of argon on the basis of polymerization. In the final polymerization, a certain amount of acetic acid was added to prevent the anion activity. The crude polymer was precipitated with $n$-hexadecane and refined twice with ether [16].

3.3. Cell Culture. After adding a low concentration of Vincetine (VCR) to non-drug-resistant cells, the concentration of Vinoxetine gradually increased from the low concentration, from $0.01 \mu \mathrm{g} / \mathrm{mL}$ to $1 \mu \mathrm{g} / \mathrm{mL}$, until the cells survived normally at this concentration. The cells were incubated at $5 \% \mathrm{CO}_{2}$ and $37^{\circ} \mathrm{C}$.

3.4. Cytotoxicity Test. The harvest was carried out in logarithmic steps and finally adjusted to 170 in RPMI-164 with $10 \%$ FBS. After 24 hours, each plate was cultured in a medium containing VCL and fg0226 or VCL and loaded with fg0226 micelles. For example, the concentration of fg0226 in the culture medium containing fg0226 or loading fg020226 miser is $2 \mu \mathrm{mol} / \mathrm{L}$. At a concentration lower than the VCC value, the cell culture of polymer was compared. The concentration gradient of VR was adjusted by medium. After 72 hours of culture, each $10 \mu \mathrm{L}$ of MTT salt solution $\left(0.9 \% \mathrm{NaCl}\right.$ saline) with a concentration of $5 \mathrm{mg} / \mathrm{M}_{1}$ was cultured on the well plate for 4 hours in a $37^{\circ} \mathrm{C}$ box. Each well was detected on $540 \mathrm{~nm}$ and $655 \mathrm{~nm}$ plates by culturing enzyme samples [17]. 
TABLE 1: Main reagents.

\begin{tabular}{lcc}
\hline Serial number & Reagent name & Origin \\
\hline 1 & Low-molecular-weight sodium hyaluronate & Huaxi Freda Biomedical Co Ltd \\
2 & Doxorubicin hydrochloride & American Sigma Company \\
3 & N-Hydroxysuccinimide (NHS) & American Sigma Company \\
4 & Tetraethyl orthosilicate (TEoS) & Sigma-Aldrich Company \\
5 & Succinyl hydrazide (SDH) & Tokyo Chemical Co Ltd \\
\hline
\end{tabular}

TABLE 2: Main instruments.

\begin{tabular}{lcc}
\hline Serial number & Instrument & Vendor \\
\hline 1 & UV-visible spectrometer & American Varian Company \\
2 & Fourier infrared diffuse reflectance spectrometer & American NEXUS Company \\
3 & Nuclear magnetic resonance system & Bruker \\
4 & X-ray spectrometer & Rigaku Corporation \\
5 & Differential thermal analyzer & PerkinElmer \\
6 & Transmission electron microscope & Hitachi \\
\hline
\end{tabular}

3.5. Mouse Wound Model. Cut wounds on the backs of healthy $\mathrm{BALB} / \mathrm{c}$ mice (6-8-week-old) and apply $106 \mathrm{CFU} /$ $\mathrm{mL}$ Staphylococcus aureus suspension that is resistant to Kobayashi Lin to the wound surface of the mice to remove bacteria to make wound infection models. Mice infected with wound bacteria were divided into 5 groups $(N=4)$, and each group used PBS buffer (control) to release CA (7.2 g/ mL). Add CA@AuMNAuMN-HA near-infrared light irradiation and CA@AuMN-HA near-infrared light for processing. Observe the wound every 24 hours, take pictures, and change the bandage. After 3 days of treatment, all mice were euthanized and wound skins were collected. Put each excised wound skin into $1 \mathrm{~mL}$ of sterile normal saline, shake to mix, collect $500 \mathrm{gL}$ of each solution, add it to $20 \mathrm{~mL}$ of liquid medium, and shake culture overnight at $37^{\circ} \mathrm{C}$, and then plate. Use a counting method to measure the number of remaining bacteria in each wound [18].

3.6. Histological Analysis. After the efficacy experiment, all the mice were dissected and killed, the main organs and tumor tissues of each group of mice were dissected, the weight of the tumor was measured, and pictures were taken. The prepared sections were used for histological analysis of serine and epoxy staining [19].

\section{Application Analysis of the Nanosystem of Optical and Chemical Therapy in the Treatment of Sports Rehabilitation Diseases}

4.1. Analysis of Hemolysis of Multifunctional Treatment Platform (RCPD). The results of hemolysis are shown in Figure 1. As the concentration increases, the hemolytic toxicity of unmodified PAMAM also increases, while RCPD has less hemolytic toxicity and good biocompatibility, which can be used for intravenous injection. Unmodified dendrimers show concentration-dependent hemolysis. Compared with unmodified dendrimers, RCPD has lower hemolysis and better biocompatibility. The lower toxicity of
RCPD than unmodified dendrimers can be explained by the different charges on its surface. The unmodified dendrimer has a high positive charge of $+12.58 \mathrm{mV}$, while the surface charge of RCPD is $+5.02 \mathrm{mV}$. The quantum size effect is caused by the size change and the formation of a tunable energy gap, the prepared nanomaterials emit light, and the optical splitter has been widely studied in the field of optics. PAMAM dendrimers cause hemolysis due to their cationic properties and the interaction with red blood cell membrane (RBC), while RCPD reduces the surface charge by modifying the surface of cypate-iRGD and reduces the interaction with red blood cell membrane. Therefore, RCPD greatly reduces the hemolytic toxicity caused by unmodified dendrimers and has better biocompatibility. Fluorescence emission spectroscopy was used to verify the feasibility of this method to detect telomerase activity by fluorescence. In order to detect telomerase activity, telomerase extracted from HeLa was incubated with TS primers in a system where deoxynucleotide mixture (dNTP) exists at $30^{\circ} \mathrm{C}$ for 1 hour to obtain the telomerase reaction product (TRP). Then, the TRP and the detection system were incubated at $37^{\circ} \mathrm{C}$ for 40 minutes, and the fluorescence intensities of different mixed systems were detected. When $\mathrm{H}_{1}$ and $\mathrm{H}_{2}$ were cocultivated with NMM, the fluorescence intensity of the system did not increase significantly compared with the NMM solution alone; that is, there was no obvious G-quadruplex production in the system, indicating that $\mathrm{H}_{1}$ and $\mathrm{H}_{2}$ were in the absence of catalyst. The chain hybridization reaction between the two is very weak. When the cocultivation system of telomerase and TS was added and incubated with $\mathrm{H}_{1}$ and $\mathrm{H}_{2}$ for a period of time and then added to the NMM solution, the fluorescence intensity was significantly enhanced, indicating that the existence of TI Chong can catalyze the rapid progress of the hybridization reaction of $\mathrm{H}_{1}$ and $\mathrm{H}_{2}$ chains. However, when telomerase is thermally denatured in advance to make it alive, the cocultivation system with TS is incubated with $\mathrm{H}_{1}$ and $\mathrm{H}_{2}$ for a period of time and then the NMM solution is added. Compared with the single NMM solution, its fluorescence intensity is not obvious. The 


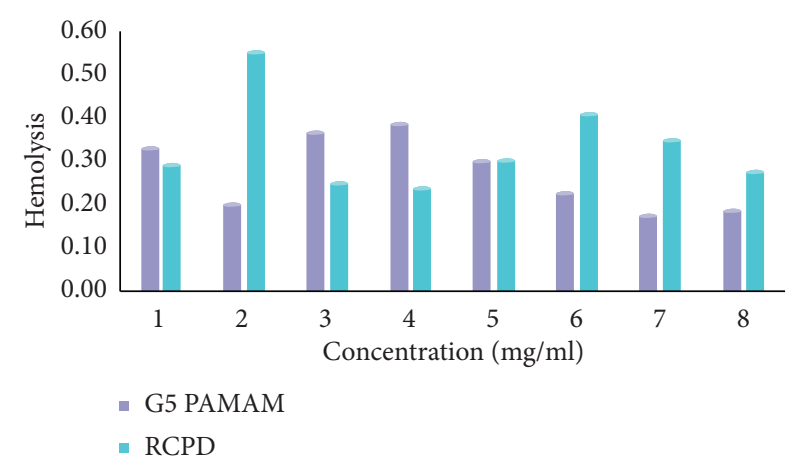

Figure 1: Hemolysis results.

changes prove that the system can indeed be used to detect different telomerase activities. The position of the drug in the micelle also affects the release. The positioning of the drug mainly depends on its interaction parameters with the hydrophobic and hydrophilic blocks. Most of the hydrophobic drugs are located in the core of the micelle, and the amphiphilic drugs are located inside the shell. And between the shell and the inner core interface, the hydrophilic drug is positioned in the shell. If the drug is mainly located in the core of the micelle, the longer the hydrophobic block and the larger the core, the slower the release rate of the drug from the micelle $[20,21]$.

The results of plate colony count are shown in Table 3 and Figure 2. The results show that CA@AuMN-HA has a better bactericidal effect than directly adding free CA. Its enhanced bactericidal effect is mainly because the existence of nanocarriers can overcome the poor water solubility and stability of CA. When the bacteria were incubated with CA@ AuMN-HA and treated with near-infrared light, the number of bacterial colonies was greatly reduced. After the bacteria are incubated with bactericidal reagents and treated with light, the activity of bacteria in different systems is determined by OD600 nanometers. It can be seen from the figure that as the concentration of the bactericidal reagent increases, its bactericidal effect is enhanced to varying degrees. However, free CA needs to be at a higher concentration to have a better killing effect. At the CA concentration contained in $80 \mu \mathrm{g} / \mathrm{mLCA} @ A u M N-H A$, free CA has only about $20 \%$ antibacterial efficiency, while CA@AuMN-HA can reach about $50 \%$ antibacterial efficiency. At the same time, the single nanocarrier AuMN-HA was incubated with the bacteria and treated with near-infrared light, and the survival rate of the bacteria decreased by $57 \%$. It is proven that the nanocarrier has a good photothermal sterilization effect. When CA@AuMN-HA is incubated with bacteria and treated with near-infrared light, the inhibition rate of bacterial activity can reach $90 \%$. This remarkable ability to inactivate bacteria proves that the CA@AuMN-HA system has excellent synergistic bactericidal ability with chemotherapy and light and heat. An important strategy for tumor immunotherapy is to destroy the tumor immunosuppressive microenvironment and induce strong T-cell response to some tumor-specific epitopes and produce continuous immune memory effects. In some cases, only the strong
T-cell response induced by specific immune adjuvants is sufficient to overcome the tumor immunosuppressive microenvironment. After the TLRs of DCs are specifically activated, they will activate the NF- $\kappa \mathrm{B}$ signaling pathway, induce the production of IL-12, and increase the expression of costimulatory receptors such as CD40. Chemotherapy uses chemical drugs to directly kill tumor cells and inhibit the growth and reproduction of tumor cells. However, these small molecule drugs have problems such as short metabolic time, poor targeting, and easy drug resistance. On the one hand, they kill tumor cells, but they also cause damage to the function of normal cells and tissues. Therefore, nanomedicine has been rapidly developed. Drug nanometerization can improve the solubility of hydrophobic drugs in water, increase the blood circulation time of drugs, enhance tumor targeting, and improve biodistribution, thereby improving the therapeutic effect. The combined use of chemotherapy and immunotherapy has a synergistic effect in tumor treatment [22].

\subsection{Characterization and Analysis of Polyhistidine} Nanomicelles. A dynamic laser particle size analyzer was used to detect and analyze the particle size, PDI, and zeta potential of PHIS. The stable particle size distribution of the system is expressed by the polydispersity index (PDI), which is a parameter obtained from the analysis of photon correlation spectroscopy. Generally speaking, the smaller the PDI, the better the uniformity of the system. Zeta potential is related to the stability of nanoparticles. It is generally believed that the zeta potential is high, the system is not easy to aggregate, and the system is stable; vice versa. The particle size, PDI, and zeta potential of PHIS with different mass ratios are shown in Table 4 and Figure 3 . When the particle size of the nanomicelle PHI formed by R848 and polyhistidine is about $150 \mathrm{~nm}$, the distribution is uniform, and the PDI is less than 0.2, indicating that the PHIS nanomicelles prepared by us have good dispersibility, and their zeta potentials are all greater than $30 \mathrm{mV}$. Through the comparison of blank micelles and micelles loaded with enzymes and prodrugs, the size of micelles after loading enzymes and prodrugs increased significantly, indicating that nanomicelles loaded with enzymes and prodrugs were successfully prepared. Among them, the outer shell is a PEG layer that stabilizes nanoparticles, which is used to stabilize polymer micelles and protect the inner core from the external environment. The second outer layer is a positively charged and reduction-sensitive polyamino acid layer, which passes through the polyamino acid side. The positively charged amino group of the chain end reacts electrostatically with the negatively charged enzyme to coat the prodrug through the hydrophobic segment to form the core of the micelle. E/H-PEAC has better cell lethality than EIA/HRP combination, which is mainly due to the protection of the enzyme and prodrug by the polymer. Furthermore, because $\mathrm{E} / \mathrm{H}-\mathrm{PEAC}$ is coated with polymer, it will be more easily taken up by tumor cells, while simple enzymes and prodrugs are not easily taken up by tumor cells. EIA and HRP are simultaneously delivered to tumor cells by micelles, and the 
TABLe 3: Plate colony count results.

\begin{tabular}{lllllll}
\hline CA & 1.2 & 1.4 & 0.8 & 1.6 & 1.7 & 2.1 \\
CA@AuMN & 0.5 & 1.1 & 0.8 & 2.4 & 1.6 & 1.2 \\
AuMN-HA & 0.4 & 1.3 & 1.5 & 2.3 & 1.6 & 1.9 \\
CA@AuMN-HA & 0.6 & 0.9 & 1.8 & 1.2 & 1.8 & 1.4 \\
\hline
\end{tabular}

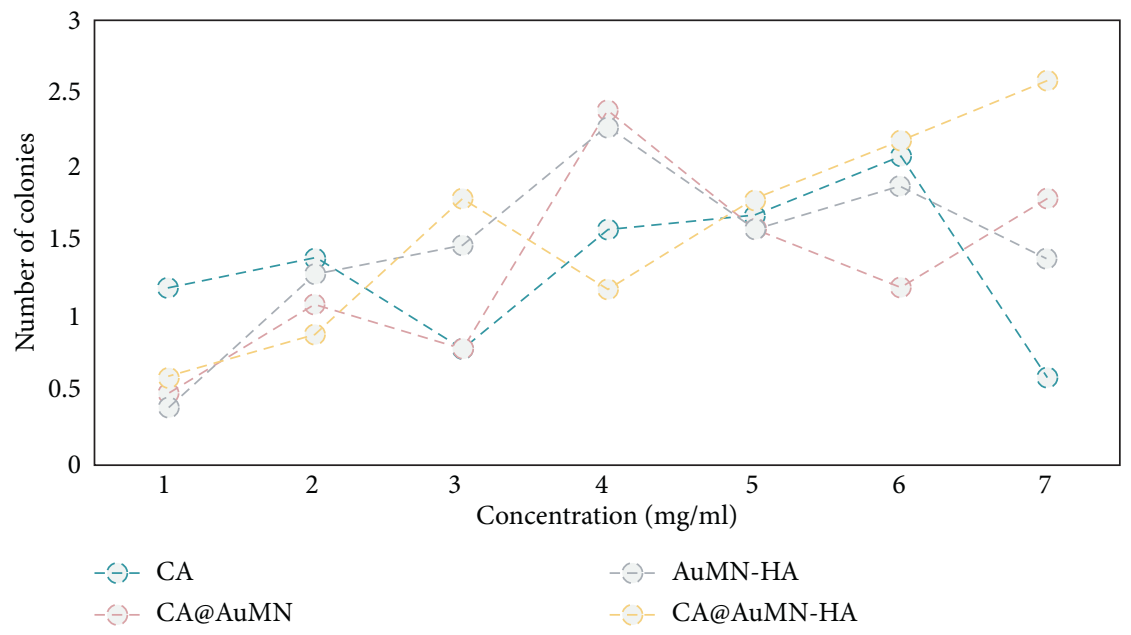

FIGURE 2: Results of plate colony count.

TABle 4: Particle size, PDI, and zeta potential of PHIS with different mass ratios.

\begin{tabular}{lccr}
\hline PHIS & Size & PDI & Zeta potential \\
\hline 1 & 138.4 & 0.122 & 37.1 \\
2 & 156.2 & 0.132 & 39.8 \\
3 & 175.6 & 0.128 & 42.7 \\
4 & 186.1 & 0.154 & 39.7 \\
5 & 145.8 & 0.227 & 32.8 \\
\hline
\end{tabular}

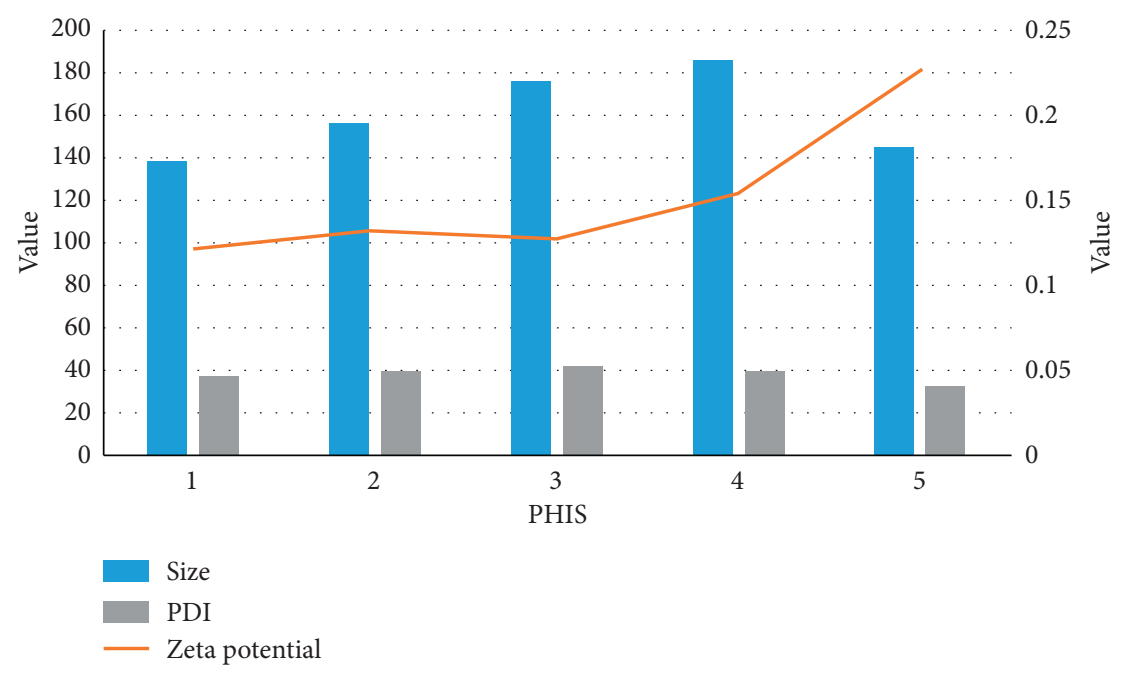

FIgURE 3: Particle size, PDI, and zeta potential of PHIS with different mass ratios. 
combination of enzymes and prodrugs produces a large amount of ROS. ROS acts on tumor cells, resulting in a faster and more effective cell killing rate. Therefore, the combined delivery of EIA and HRP has great potential in cancer treatment. As a minimally invasive light-stimulated therapy mode, photodynamic therapy has been clinically approved and has been successfully used to treat esophageal cancer, skin cancer, and non-small-cell lung cancer. Photodynamic therapy refers to the photosensitizer enriched in the lesion site in an aerobic environment, activated by laser irradiation of a specific wavelength, and then transfers the energy to the surrounding oxygen to generate reactive oxygen species (ROS), thereby killing tumor cells. ROS includes peroxides, singlet oxygen, and oxygen-containing free radicals. They are highly reactive and can cause apoptosis or necrosis through cellular oxidative stress. The main principle of photodynamic therapy (PDT) is that the photosensitizer absorbs light energy to produce energy level transitions and releases cytotoxic reactive oxygen species, which can further cause tumor cell apoptosis or necrosis. Sports rehabilitation refers to the use of various means of sports to recover and reconstruct the functions that have been lost by the sick, injured, and disabled as soon as possible and as much as possible. It is an integral part of rehabilitation medicine. Sports rehabilitation is the development and extension of exercise therapy. It is the use of sports methods and various sports methods to treat diseases and injuries, prevent complications, relieve negative mental states, promote the full recovery of physical functions, and achieve the purpose of rehabilitation $[23,24]$.

4.3. Analysis of Cell Viability Results. The results of the cytotoxicity test are shown in Figure 4. The MTT method was used to investigate the cytotoxicity of different concentrations of CLB and CLB-HDH micelles to K562 cells, MCF-7 cells, and B16F10 cells. The experimental results are shown in Figure 4. Within the scope of the study, the inhibition rate of CLB and CLB-HDH micelles on tumor cells both showed concentration-dependent and time-dependent. With the increase in concentration and time, the cell inhibition rate continued to increase. After $48 \mathrm{~h}$ of incubation under the same drug dosage condition, the cell inhibition rate of CLB$\mathrm{HDH}$ micelles was significantly higher than that of CLB. After $24 \mathrm{~h}$ or $48 \mathrm{~h}$ incubation with the cells, CLB-HDH micelles have lower IC50 values than CLB. At the same dose, CLB-HDH micelles have a higher killing rate to tumor cells than CLB. The normal physiological environment was simulated in vitro, and the release behavior of free CLB molecules and CLB-HDH micelles was studied under the same conditions by dialysis. The cumulative release of free CLB molecules within $3 \mathrm{~h}$ almost reached $100 \%$, and its rapid release can be attributed to its small molecular size. In contrast, the release amount of CLB-HDH micelles within $3 \mathrm{~h}$ is $62 \%$, which has a certain slow-release effect. In tumor cells, micelles are unassembled, and CLB-HDH molecules are decomposed into CLB under the action of lysosomal proteases. The surface of nanomaterials is different from the internal lattice symmetry of nanostructures (tetrahedral and octahedral symmetry). Therefore, the chemical environment and boundaries of the surface atoms of nanomaterials are different from those inside the nanomaterials. The surface atoms have unpaired chemical bonds and unpaired electrons. The donor or acceptor states can be formed on the surface, which exists in the band gap. The surface atoms of nanomaterials do not have stable symmetry. The surface atoms have high energy and are easy to adsorb molecules in the air, causing the electronic structure to change. Using this adsorption characteristic, nanogas sensors can be prepared. Nanowire materials with a high surface activity use the surface area of nanomaterials to increase the reaction rate and photocatalytic efficiency. This is an unattainable advantage of bulk materials. However, it is precise because the surface unpaired electrons cause the surface state and charge accumulation, which causes the surface energy band to bend, which causes the transport carriers and the surface state to scatter, and reduces the carrier mobility of nanomaterial devices. As the size decreases, the area of nanomaterials increases, and the stability of nanostructures decreases. In addition, nanodrug carriers can deliver drugs to tumor tissues through two targeting mechanisms, passive targeting, and active targeting, which is beneficial to increase the concentration of drugs at tumor sites and reduce the side effects of drugs on normal tissues. Under ideal circumstances, as long as the drug reaches an effective therapeutic concentration in the body, it can target cells to treat diseases. However, drug delivery is difficult to control. It is difficult to control the release rate of drugs, the specific targeting of cells or tissues, and drug stability. In order to solve this difficulty, the researchers used various materials and chemistry strategies to design various drug delivery systems. The drug delivery system can improve the specificity of drug treatment by enhancing the stability of the drug in the body, controlling the release of the drug, and localizing the action of the drug. Many materials can extend the circulation time of drugs in the body or accumulate and release drugs at target sites in the body. The drug delivery system can adjust the distribution of drugs in the body as needed. The development of synthetic chemistry, materials science, medicinal chemistry, and conjugate chemistry has promoted the development of drug delivery systems, and drug delivery systems are becoming more and more common in clinical applications. Due to the emergence of nucleic acids, antibodies, proteins, and conjugated drugs, the medical field is actively changing. The modified drug molecule may be several orders of magnitude larger than the original small drug molecule, and it is also more sensitive to the environment, so it needs proper protection and its biocompatibility and specificity. Therefore, the drug delivery system needs further development [25]. 


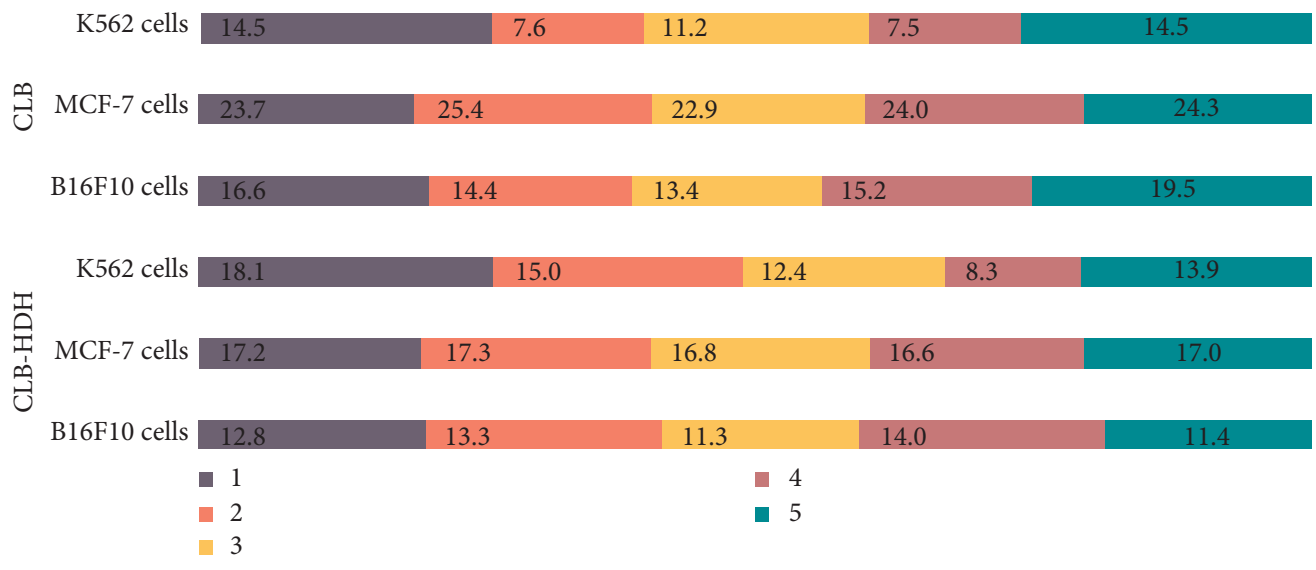

Figure 4: Cytotoxicity test results.

\section{Conclusions}

This article mainly studies the application of nanosystems combined with optics and chemotherapy in the treatment of sports rehabilitation diseases. We have synthesized a multifunctional treatment platform RCPD, which integrates phototherapy, chemotherapy, and image formation functions through amidation reaction. Hydrophobic CLB and hydrophilic $\mathrm{HDH}$ were covalently connected by amide bond, and the amphiphilic prodrug CLB-HDH was synthesized. Th successful synthesis was proved by proton nuclear magnetic resonance spectroscopy and mass spectrometry. When CLB-HDH was dispersed in water, CLB$\mathrm{HDH}$ self-assembled into spherical micelles with a diameter of $49 \mathrm{~nm}$ and aggregated in tumor sites targeting the EPR effect during circulation in vivo. Fluorescence bottom-up microscopy showed that CLB-HDH micelles were effectively absorbed by tumor cells. The cytotoxicity test showed that CLB-HDH micelles had a stronger cytotoxicity than free CLB molecules. The hemolysis test showed that CLB-HDH micelles could be injected intravenously. The results of the in vivo tumor inhibition test and histological analysis showed that CLB-HDH micelles could significantly inhibit tumor growth with good biological safety and low systemic toxicity. CLB-HDH micelles provide a new idea for the effective treatment of tumor by CLB.

The size of the nanomaterials is $10-100 \mathrm{~nm}$, which is absorbed by the cells through the terminal nervous system, and is concentrated on the tumor site through the EPR effect, achieving the goal of passive tumors and the improvement in drug utilization. Mesoporous silica nanoparticles (MSNs) are a new type of inorganic nanomaterials with high biocompatibility, large surface area, controllable size, and easy surface modification. They are widely used as carriers of antitumor drugs. The effective concentration of the drug can be increased by loading the drug on the MSN or adsorbing it on the surface.

Compared with the drug delivery system that uses tumor cell membrane modification, the tumor incidence is good. Compared with a single treatment mode, the combined therapy combining the chemotherapy effect of DOX and the photothermal effect of ICG can improve the killing effect of tumor cells. Using the nanosystem, the detection of endogranular enzyme activity can be reduced to the level of single cell extracts. In addition, the system sensitively distinguishes multiple types of cancer cell lines with different endogranzyme activities and is used to effectively screen endogranzyme inhibitors. In addition, if the concept of DAN fuel molecular machinery is introduced into the detection system, the cycle process can be realized without the catalytic effect of added nucleotides, and the fluorescence signal can be greatly increased.

\section{Data Availability}

The data in this article are available from the corresponding author on reasonable request.

\section{Disclosure}

The authors confirm that the content of the manuscript has not been published or submitted for publication elsewhere.

\section{Conflicts of Interest}

The authors declare that there are no potential conflicts of interest in our paper.

\section{Authors' Contributions}

All authors have seen and approved the manuscript.

\section{Acknowledgments}

This study was supported by the National Social Science Fund of China (no. 17BTY075, Research on the Innovation of Sports Activities in Primary and Secondary Schools in China under the Background of Healthy China) and Philosophy and Social Science Fund of Hunan Province (no. 19YBA124, Innovative Research on Sports Activity Pattern of Primary and Secondary Schools in Hunan Province under the Background of Sports Development). 


\section{References}

[1] A. V. Soares, N. G. Borges Jr., M. Hounsell, E. Marcelino, G. M. Rossito, and Y. Sagawa Jr., "A serious game developed for physical rehabilitation of frail elderly," European Research in Telemedicine, vol. 5, no. 2, pp. 45-53, 2016.

[2] L. G. Spagnoli, A. Mauriello, Y. Sambuy et al., "Interaction detection with depth sensing and body tracking cameras in physical rehabilitation," Methods of Information in Medicine, vol. 55, no. 1, pp. 70-78, 2016.

[3] O. Dana, L. Jimmy, Y. Fan, B. Stephen, and Y. Joshua, "Activity-based physical rehabilitation with adjuvant testosterone to promote neuromuscular recovery after spinal cord injury," International Journal of Molecular Sciences, vol. 19, no. 6, p. 1701, 2018.

[4] D. L. Dycus, D. Levine, and D. J. Marcellin-Little, "Physical rehabilitation for the management of canine hip dysplasia," Veterinary Clinics of North America: Small Animal Practice, vol. 47, no. 4, p. 823, 2017.

[5] S. Vedala and M. Sushama, "Urea assisted combustion synthesis of $\mathrm{LiFePO} 4 / \mathrm{C}$ nano composite cathode material for lithium ion battery storage system," Materials Today: Proceedings, vol. 5, no. 1, pp. 1649-1656, 2018.

[6] D. J. Mcwilliams, S. Benington, and D. Atkinson, "Outpatientbased physical rehabilitation for survivors of prolonged critical illness: a randomized controlled trial," Physiotherapy Theory and Practice, vol. 32, no. 3, pp. 179-190, 2016.

[7] O. Lazareva, M. Aravitska, O. Andrieieva et al., "Dynamics of physical activity status in patients with grade I-III obesity in response to a physical rehabilitation program," Journal of Physical Education \& Sport, vol. 17, no. 3, pp. 1960-1965, 2017.

[8] N. M. Salbach, S. Wood-Dauphinee, J. Desrosiers et al., "Facilitated interprofessional implementation of a physical rehabilitation guideline for stroke in inpatient settings: process evaluation of a cluster randomized trial," Implementation Science, vol. 12, no. 1, p. 100, 2017.

[9] R. Unhjem, G. Flemmen, J. Hoff, and E. Wang, "Maximal strength training as physical rehabilitation for patients with substance use disorder; a randomized controlled trial," Bmc Sports Science Medicine \& Rehabilitation, vol. 8, no. 1, pp. 1-10, 2016.

[10] D. Jia, S. Z. Xu, S. Guo, and Y. Shen, "Practical research on physical rehabilitation training among male addicts in Chinese compulsory isolated detoxification center," Open Journal of Preventive Medicine, vol. 8, no. 4, pp. 121-130, 2018.

[11] W. I. Baltzer, S. Smith-Ostrin, J. J. Warnock, and C. G. Ruaux, "Evaluation of the clinical effects of diet and physical rehabilitation in dogs following tibial plateau leveling osteotomy," Journal of the American Veterinary Medical Association, vol. 252, no. 6, pp. 686-700, 2018.

[12] R. A. H. M. Alalwan, V. Vitomskiy, O. Lazareva, and M. Vitomska, "Physical rehabilitation after achilles tendon ruptures: a review of modern approaches," vol. 58, no. 2, pp. 78-86, 2017.

[13] M. Shirazikhah, H. A. Mirabzadeh, A. BiglarianJoghataei et al., "National survey of availability of physical rehabilitation services in Iran: a mixed methods study," Electronic Physician, vol. 9, no. 11, pp. 5778-5784, 2017.

[14] D. Mohammadi Shahboulaghi, Ó. González-Castán, D. Huepe, and D. Huepe, "From ancient Greece to the cognitive revolution: a comprehensive view of physical rehabilitation sciences," Physiotherapy Theory and Practice, vol. 33, no. 2, pp. 89-102, 2017.
[15] Z. Nourmohammadi, A. Khalifehzadeh-Esfahani, M. Eftekhari et al., "The effect of aerobic physical rehabilitation on the quality of life in patients with chronic atrial fibrillation; a randomized controlled clinical trial study," ARYA Atherosclerosis, vol. 15, no. 1, pp. 1-8, 2019.

[16] T. A. Olugbade, A. Singh, N. Marquardt, N. B. B. Berthouze, M. S. H. Aung, and A. C. D. C. Williams, "How can affect be detected and represented in technological support for physical rehabilitation?" ACM Transactions on Computer-Human Interaction, vol. 26, no. 1, pp. 1-29, 2019.

[17] T. S. WilliamsAung, M. D. Landry, H. Hoenig et al., "Physical rehabilitation needs in the BRICS nations from 1990 to 2017: cross-national analyses using data from the global burden of disease study," International Journal of Environmental Research and Public Health, vol. 17, no. 11, p. 4139, 2020.

[18] G. KamalakannanZeng, D. Khorasani-Zavareh, A. Ardalan et al., "Continuous post-disaster physical rehabilitation: a qualitative study on barriers and opportunities in Iran," Journal of Injury \& Violence Research, vol. 11, no. 1, pp. 35-44, 2019.

[19] M. Shirazikhah, A. Mirabzadeh, H. Sajadi et al., "Development and psychometric properties of the physical rehabilitation services acceptability questionnaire," Medical Journal of the Islamic Republic of Iran, vol. 33, no. 1, pp. 202-209, 2019.

[20] N. P. Lyamina and E. S. Karpova, "Concept of choosing of physical rehabilitation programs for patients with coronary artery disease," Cardiovascular Therapy and Prevention, vol. 18, no. 2, pp. 13-19, 2019.

[21] T. Odynets and Y. Briskin, "Effect of individualised physical rehabilitation programmes on the functional state of the cardiovascular system in women with post-mastectomy syndrome," International Journal of Therapy and Rehabilitation, vol. 26, no. 2, pp. 1-10, 2019.

[22] A. Nogas, I. Grygus, O. Nagorna et al., "Results of the physical rehabilitation of patients with pulmonary tuberculosis," Journal of Physical Education and Sport, vol. 19, no. 1, pp. 684-690, 2019.

[23] L. I. Vasilyeva, E. D. Egudina, O. S. Kalashnikova, I. A. Zhivilo, and L. V. Sapozhnichenko, "Physical rehabilitation of patients with pulmonary hypertension," Voprosy Kurortologii, Fizioterapii I Lechebnoi Fizicheskoi Kul'tury, vol. 96, no. 5, pp. 51-60, 2019.

[24] T. Zhivilo, M. Landry, and H. Hoenig, "Global need for physical rehabilitation: systematic analysis from the global burden of disease study 2017," International Journal of Environmental Research and Public Health, vol. 16, no. 6, p. 980, 2019.

[25] D. Wynne and K. Cooper, "Student led physical rehabilitation groups and clinics in entry level health education: a scoping review protocol," JBI Database of Systematic Reviews and Implementation Reports, vol. 17, no. 6, pp. 1092-1100, 2019. 